\title{
Metamaterial-inspired Electrically Small Platforms: Enhanced Directivity Properties
}

\author{
Richard W. Ziolkowski ${ }^{1,2}$ \\ (1) Global Big Data Technologies Centre, University of Technology Sydney, \\ Ultimo NSW 2007, Australia \\ (2) The University of Arizona, Tucson, AZ 85721, USA
}

\begin{abstract}
A variety of near-field resonant parasitic (NFRP) antennas have been developed as electrically small platforms to realize high directivity. These include compact arrays and Huygens dipole and multipole radiating systems. A brief review of these developments and their scattering equivalents will be presented.
\end{abstract}

Keywords-Dipole antennas; electrically small antennas; Huygens antennas; multipole antennas; near-field resonant parasitic antennas

\section{INTRODUCTION}

With the introduction of metamaterials and metamaterialinspired constructs, a number of advances in the performance of electrically small (ES) radiators and scatterers in the VHF, UHF, microwave, millimeter-wave, and optical regimes have been achieved. Many of these platforms have been based on resonant near-field parasitic (NFRP) designs. They have been fabricated and tested; these measurement results are in nice agreement with predictions [1].

There are a variety of applications for which it is desirable to have higher directivity and/or a large front-to-back ratio (FTBR), i.e., to have the radiated power emitted primarily into one hemisphere. Several NFRP designs have explored the ability to achieve higher directivity from ES platforms. These include the use of electromagnetic band gap (EBG) structures as structured ground planes [2], [3] and compact arrays [4]. Combinations of electric and magnetic multipole NFRP elements have led to ES Huygens dipole [5]-[11] and multipole systems [13]. Additional augmentations have produced efficient, broad-bandwidth, high directivity NFRP ESAs [14].

\section{COMPACT ARRAY}

An Egyptian axe (electric) dipole (rectangular version) and a 3D magnetic EZ antenna were combined into a compact array to study its directivity properties [4]. This configuration is shown in Fig. 1(a). It was found that by overlapping the resonance frequencies of both antennas; driving the electric element and leaving the magnetic element passive; and matching their phase centers, one could achieve an efficient, ES system with high directivity and FTBR system.

The 3D directivity pattern of this $k a=0.407$ array shown in Fig. 1(b) emphasizes this outcome. The peak FTBR value is $510.54(27.0 \mathrm{~dB})$ at $301.154 \mathrm{MHz}$, while the radiation efficiency is $\mathrm{RE}=95.31 \%$. At that frequency, the accepted and total radiated powers are, respectively, $0.949 \mathrm{~W}$ and 0.904 W. These results clearly demonstrate that a broadside cardioid pattern has been generated efficiently by this array.

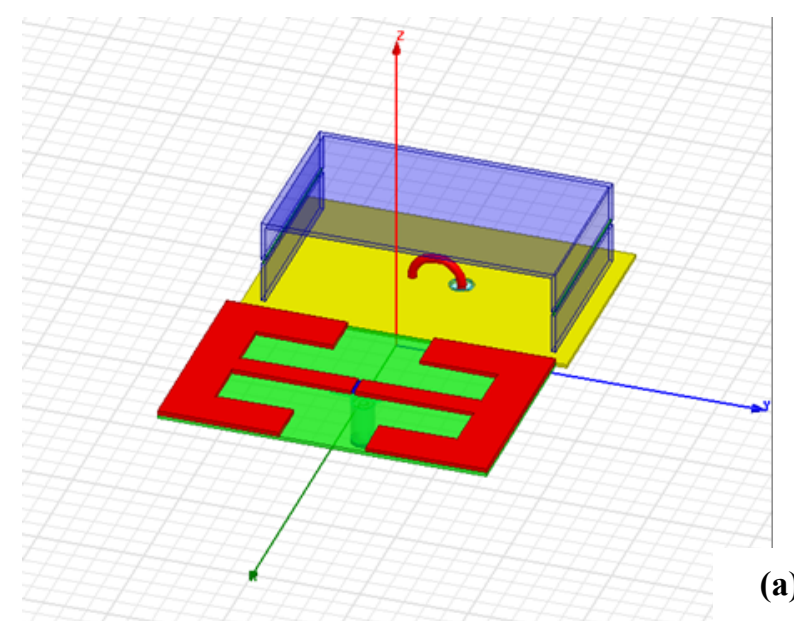

(a)
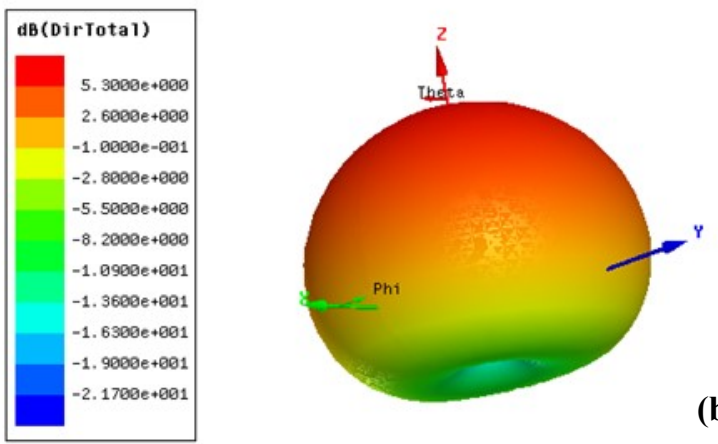

(b)

Fig. 1 Two element, ES compact array consisting of an electric and magnetic NFRP antenna, the electric one is driven by a $50 \Omega$ source and the magnetic one is passive and matched to $50 \Omega$. (a) Configuration. (b) Directivity. [4]

\section{HUYGENS ANTENNAS}

The application of a high directivity, broadside radiating, conformal antenna has many current and future wireless platform applications. Various successful designs have been confirmed [5]-[12]. For instance, a $28 \mathrm{GHz}$ version of linearly polarized (LP) Huygens dipole antenna has been recently 
reported [11]. Its CP version was only designed and simulated in that work. A CP design [12], as shown in Fig. 2, operating at $1.58 \mathrm{GHz}$ was simulated, fabricated, assembled, and measured successfully. The realized antenna is compact in size $(k a=$ $0.87)$, low-profile $\left(\sim 0.04 \lambda_{0}\right)$, broadside radiating with a large FTBR $(>20 \mathrm{~dB})$, and has a high radiation efficiency (RE $>$ 93.9\%) at its resonance frequency. The balun viewed in Fig. 2 is present solely for measurement purposes to mitigate the effects of any spurious cable currents.

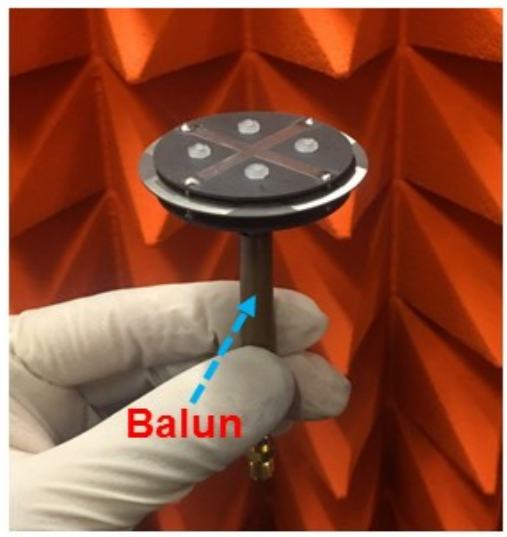

Fig 2 Measured, low profile, broadside radiating, CP Huygens dipole antenna prototype [12].

\section{CONCLUSION}

A variety of NFRP antennas that have been demonstrated to produce Huygens source cardioid patterns will be presented at the conference. Their operating principles will be discussed. Ongoing efforts to produce yet higher directivities with Huygens multipole metamaterial-inspired constructs [13] and broader bandwidths with ES NFRP Huygens dipole antennas augmented with non-Foster elements [14] will be shared. Related metamaterial-inspired NFRP scattering geometries in the optical regime will demonstrate their ability to transduce diverging source fields into highly directive beams.

\section{REFERENCES}

[1] R. W. Ziolkowski, P. Jin, and C.-C. Lin, "Metamaterial-inspired engineering of antennas," Proc. IEEE, vol.99, no.10, pp.1720-1731,Oct. 2011.

[2] P. Jin, and R. W. Ziolkowski, "High-directivity, electrically small, lowprofile near-field resonant parasitic antennas," IEEE Antennas Wirel. Propag. Lett., vol. 11, pp. 305-309, 2012.

[3] S. Chen, W. Lin, R. W. Ziolkowski and Y. J. Guo, "Reconfigurable, wideband, low-profile, circularly polarized antenna and array enabled by an artificial magnetic conductor ground," accepted, IEEE Trans. Antennas Propag., 01 Jan. 2018.

[4] R. W. Ziolkowski, "The directivity of a compact antenna: An unforgettable figure of merit," EPJ Appl. Metamat., vol. 4, 7, 2017.

[5] P. Jin and R. W. Ziolkowski, "Metamaterial-inspired, electrically small, Huygens sources," IEEE Antennas Wireless Propag. Lett., vol. 9, pp. 501-505, May 2010.

[6] R. W. Ziolkowski, M.-C. Tang and N. Zhu, "An efficient, broad bandwidth, high directivity, electrically small antenna," Microw. Opt. Technol. Lett., vol. 55, no. 6, pp. 1430-1434, June 2013.

[7] I. Liberal, I. Ederra, R. Gonzalo, and R. W. Ziolkowski, "Induction theorem analysis of resonant nanoparticles: Design of a Huygens source nanoparticle laser," Phys. Rev. Applied, vol. 1, 044002, May 2014.

[8] R. W. Ziolkowski, "Low profile, broadside radiating, electrically small Huygens source antennas," IEEE Access, vol. 3, pp. 2644-2651, Dec. 2015.

[9] M.-C. Tang, H. Wang, and R. W. Ziolkowski, "Design and testing of simple, electrically small, low-profile, Huygens source antennas with broadside radiation performance," IEEE Trans. Antennas Propag., vol. 64, no. 11, pp. 4607-4617, Nov. 2016.

[10] M.-C. Tang, B. Zhou, and R. W. Ziolkowski, "Low-profile, electrically small, Huygens source antenna with pattern-reconfigurability that covers the entire azimuthal plane," IEEE Trans. Antennas Propag., vol. 65, no. 3, pp. 1063-1072, Mar. 2017.

[11] M.-C. Tang, T. Shi, and R. W. Ziolkowski, "A study of $28 \mathrm{GHz}$, planar, multi-layered, electrically small, broadside radiating, Huygens source antennas," IEEE Trans. Antennas Propag., vol. 65, pp. 6345-6354, Dec. 2017

[12] W. Lin and R. W. Ziolkowski, "Electrically-small, low-profile, Huygens circularly polarized antenna," IEEE Trans. Antennas Propag., early access, 20 Dec. 2017, DOI: 10.1109/TAP.2017.2784432.

[13] R. W. Ziolkowski, "Huygens multipole arrays to realize unidirectional needle-like radiation," Phys. Rev. X, vol. 7, 031017, Jul. 2017.

[14] M.-C. Tang, T. Shi, and R. W. Ziolkowski, "Electrically small, broadside radiating Huygens source antenna augmented with internal non-Foster elements to increase its bandwidth," IEEE Antennas Wirel. Propag. Lett., vol. 16, pp. 712-715, 2017. 